\title{
Drop-out in der Weiterbildung - eine Verschränkung von Perspektiven zur (Re-)Konstruktion des Phänomens Drop-out
}

\author{
Stefanie Hoffmann (D) • Veronika Thalhammer • Aiga von Hippel • \\ Bernhard Schmidt-Hertha
}

Eingegangen: 23. April 2019 / Angenommen: 21. August 2019 / Online publiziert: 6. September 2019 (C) Der/die Autor(en) 2019

Zusammenfassung Die vorzeitige Beendigung von Bildungsmaßnahmen wird i.d.R. als Abbruch problematisiert. Zum Problem werden Weiterbildungsabbrüche besonders dann, wenn vorhandene Weiterbildungsinteressen nicht weiter realisiert werden können und/oder wenn damit ein grundsätzlicher Rückzug aus dem System Weiterbildung eingeleitet wird. In der deutschsprachigen Bildungsforschung (v. a. in der Hochschul-, Schul- und Berufsbildungsforschung) etabliert sich zunehmend der bereits weit verbreitete Begriff „Drop-out“ als Bezeichnung für den Abbruch einer Bildungsteilnahme. In der Weiterbildungsforschung ist dieser Begriff jedoch weniger geläufig und es fehlen bisher einschlägige Studien zu Ursachen und Stellenwert des Phänomens Drop-out. Vor diesem Hintergrund widmen sich die Autorinnen und Autoren dem bestehenden Bedarf an Grundlagenforschung und nehmen eine systematische Verortung des Phänomens im Kontext von Weiterbildung vor. Dazu erarbeiten sie zunächst, welches Verständnis von Drop-out in der Disziplin der Erwachsenenbildung gängig ist. Darüber hinaus verschränken sie den aktuellen

S. Hoffmann, M.A. $(\bowtie) \cdot$ Prof. Dr. A. von Hippel Humboldt-Universität zu Berlin, Berlin, Deutschland E-Mail: s.hoffmann@hu-berlin.de

Prof. Dr. A. von Hippel

E-Mail: aiga.von.hippel@hu-berlin.de

Dr. V. Thalhammer · Prof. Dr. B. Schmidt-Hertha

Eberhard Karls Universität Tübingen, Tübingen, Deutschland

Dr. V. Thalhammer

E-Mail: veronika.thalhammer@uni-tuebingen.de

Prof. Dr. B. Schmidt-Hertha

E-Mail: bernhard.schmidt-hertha@uni-tuebingen.de 
Stand der Forschung mit Perspektiven von elf $\mathrm{zu}$ dieser Thematik interviewten Vertreterinnen und Vertretern von Weiterbildungsträgern und -verbänden. Vertieft werden diese Erkenntnisse durch eine Reflexion der Operationalisierung von Weiterbildungsabbrüchen im Nationalen Bildungspanel (NEPS). In einem Fazit werden die Ergebnisse aus Empirie und Theorie verdichtet und Implikationen für weitere (empirische) Forschungen aufgezeigt.

Schlüsselwörter Weiterbildungsabbruch · Drop-out · Weiterbildungsverhalten · NEPS · Konstellationen · Experteninterviews

\title{
Drop-out in further education-a linking of perspectives for the (re-)construction of the drop-out phenomenon
}

\begin{abstract}
The early termination of educational measures is usually critically discussed as "drop-out". The discontinuation of further education activities is considered a problem above all when existing interest in further education cannot be realized and/or when this initiates a categorical withdrawal from the system of further education. In educational research carried out in German-speaking countries (especially in research on university-, school-, and vocational education), the already wide-spread term "drop-out" is increasingly used as a synonym for the discontinuation of educational participation. In research on further education, however, this term is less common and, so far, no relevant studies have been conducted regarding the reasons for dropping out, the methods of dealing with this issue, or the importance of the drop-out phenomenon in continuing education. Against this background, the authors focus on the actual need for basic research and carry out a systematic categorization of the drop-out phenomenon in the context of further education. To this end, they first investigate which drop-out concept is most common in the field of adult education. They then link the present state of the art in research to perspectives formulated by eleven experts (representatives of education providers and continuing education associations) interviewed with regard to this topic. Findings are further substantiated through a reflection on the operationalization of early termination of further education in the National Educational Panel Study (NEPS). In a discussion summarizing the major lines of reasoning, the results thus gained are condensed against the theoretical and empirical background and guiding implications for future (empirical) research activities are outlined.
\end{abstract}

Keywords Drop-out · Adult education - Educational behaviour · Panel study · Constellation $\cdot$ Expert interviews

\section{Einleitung}

Der Abbruch von Bildungsaktivitäten wird allgemein als problematisch verhandelt, insofern besonders im formalen Bildungsbereich erst mit Erreichen des jeweiligen Abschlusses Bildungsprozesse als erfolgreich gesehen werden. Aber auch für nicht mit einem anerkannten Zertifikat verbundene Bildungsangebote ist ein vorzeitiger 
Abbruch kritisch zu sehen. Es stellt sich weiterführend die Frage, inwieweit die Bildungsteilnahme auf eine mangelnde Qualität des Angebots, unklare Information über das Angebot bzw. unzureichende Beratung im Vorfeld oder eine Verlagerung von Lerninteressen im Zeitablauf hindeutet. Zudem ist über die individuellen und organisationalen Folgen von Weiterbildungsabbrüchen - die hier unter dem Stichwort „Drop-out“ diskutiert werden - wenig bekannt. Diese Bereiche stellen - insbesondere in der Erwachsenen- und Weiterbildung - Forschungsdesiderate dar.

Drop-out in Bildungsprozessen sowie dessen Einflussfaktoren und Folgen wurden in schulischen Kontexten (z. B. Stamm et al. 2012) und in der beruflichen Bildung (z. B. Gaupp et al. 2011) in den vergangenen Jahrzehnten immer wieder empirisch beobachtet und theoretisch aufgegriffen. Aktuell laufen im Rahmen eines Förderschwerpunkts zahlreiche Forschungsarbeiten zu Drop-outs in der Hochschule. ${ }^{1}$ Die Ergebnisse zeigen übergreifend, dass Drop-out keine kurzfristige Entscheidung darstellt, sondern meist durch vorhergehendes Fernbleiben bzw. eine Distanz gekennzeichnet ist, multikausal bedingt wird und dessen Konstellationen heterogen sind. Speziell für den Bereich der Erwachsenenbildung gab es nach vereinzelten Arbeiten in den 1980er Jahren jedoch nur wenige Versuche, Weiterbildungsabbrüche empirisch $\mathrm{zu}$ untersuchen und theoretisch zu fassen.

Im Zentrum dieses Beitrags ${ }^{2}$ stehen die begrifflich-inhaltliche Klärung von Dropout sowie die Vorstellung erster Ergebnisse eines Forschungsprojekts, welches das Ziel verfolgt, Drop-out als eine Form von Weiterbildungsverhalten zu betrachten und diese Perspektive im Kontext von Theorien zur Weiterbildungsbeteiligung zu verorten. Ziel dieses Beitrags ist es daher, sich dem Phänomen Drop-out und seiner Lesart in Erwachsenenbildungskontexten empirisch zu nähern, wobei eine Expertenbefragung einerseits und die Operationalisierung von Ergebnissen aus Untersuchungen zu Weiterbildungsabbrüchen im Nationalen Bildungspanel (NEPS) andererseits herangezogen werden. Als Leitfragen für diesen Beitrag ließen sich folgende formulieren: Wie lässt sich das Phänomen Drop-out für den Bereich der Erwachsenenbildung begrifflich schärfen? Welches Verständnis herrscht hierzu im Feld der Erwachsenenbildung sowie in der Erwachsenenbildungsforschung vor?

In diesem Zusammenhang wird zunächst ein Überblick über bislang vorliegende Arbeiten und Theoriebezüge zu diesem Thema gegeben, bevor eigene empirische Daten vorgestellt werden.

\section{Der Begriff „Drop-out““ in der Erwachsenenbildung}

Im erziehungswissenschaftlichen Kontext ist der Begriff „Drop-out“ v.a. im primären, sekundären und tertiären Bildungsbereich geläufig. Es wird Folgendes darunter verstanden: „Schüler und Studierende, die einen begonnenen Bildungsweg vorzeitig ohne Abschluss aus unterschiedlichen Gründen beenden (Abbrecher)“

\footnotetext{
1 https://www.wihoforschung.de/studienerfolg-und-studienabbruch-28.php.

2 Für kritische Anmerkungen während des Schreibprozesses danken wir besonders Dr. Maria Stimm, wissenschaftliche Mitarbeiterin des Lehrstuhls Erwachsenenbildung/Weiterbildung am Institut für Erziehungswissenschaften der Humboldt-Universität zu Berlin.
} 
(Tenorth und Tippelt 2007, S. 168). ${ }^{3}$ Im deutschsprachigen Diskurs der Erwachsenenbildung wird der Begriff „Drop-out“ nur selten verwendet (vgl. Schmidt 2011, S. 203). Vor diesem Hintergrund gilt es zu ergründen, inwiefern der Begriff „Dropout" auch im Erwachsenenbildungskontext von Bedeutung ist.

Wird der Begriff verwendet, so wird damit in einem weiten Verständnis der „Abbruch einer begonnenen Weiterbildungsaktivität“ (ebd., S. 203) oder in einem engeren Verständnis der „Abbruch eines Kurses“ (Gruber 1985a, S. 3) bezeichnet. Für das Phänomen Drop-out werden in der erwachsenenpädagogischen Literatur verschiedene Bezeichnungen angewendet. Diese werden häufig synonym verwendet, haben aber strenggenommen nicht die gleiche Bedeutung (vgl. Gruber 1985a, S. 3):

1. Kursabbruch/Vorzeitiger Abgang: In dieser Bezeichnung wird auf das Verhalten von Personen Bezug genommen, welche ,die Teilnahme an einer bestimmten Erwachsenenbildungsveranstaltung (=Kurs), deren Beginn und Ende genau festgelegt sind, vorzeitig aufgeben“ (Gruber 1985a, S. 3).

2. Wegbleiben: Mit der Bezeichnung „Wegbleiber“ als Gegensatz zu „Dableiber“ wird hervorgehoben, dass Drop-out durch ein räumliches Fernbleiben der Teilnehmerin bzw. des Teilnehmers von einem Kurs gekennzeichnet ist (vgl. Schröder 1976, S. 15; Nuissl 2010, S. 69). Zu beachten ist hierbei, dass eine unregelmäßige Teilnahme davon abgrenzend als ,Teilnehmerfluktuation“ bezeichnet wird (vgl. Schrader 1986, S. 39).

3. Teilnehmerschwund: Überwiegend in empirischen Arbeiten der Erwachsenenbildungsforschung wird zur Bezeichnung des Drop-out-Phänomens der Terminus „Teilnehmerschwund“ verwendet. Damit wird die Differenz zwischen der Anzahl der angemeldeten und der Anzahl der regelmäßig Teilnehmenden fokussiert. Hier werden auch Personen einbezogen, die weder zum Kursbeginn erscheinen noch zu irgendeinem Zeitpunkt am Kurs teilnehmen (vgl. ebd., S. 38-39).

Diesen verschiedenen Termini ist letztendlich gemeinsam, dass sie ein Phänomen beschreiben, bei welchem Personen, die zu einer Weiterbildungsmaßnahme angemeldet sind und bis zu einem bestimmten Zeitpunkt an ihr teilnehmen, ihre Teilnahme vor regulärem Ende dieser Maßnahme einstellen.

\section{Theoretische Perspektiven auf Drop-out als Weiterbildungsverhalten}

Bei den Betrachtungen von Drop-out erscheint der Blick auf theoretische Ansätze der Forschung zu Adressatinnen und Adressaten, bei denen es um Motive und Barrieren sowie um das Zustandekommen von Beteiligung geht, naheliegend und fruchtbar. Diese Aspekte werden auch hier im Kontext von (1) theoretischen Ansätzen zum Weiterbildungsverhalten allgemein und (2) spezifisch zu Drop-out verfolgt.

\footnotetext{
3 Für Ausführungen zu Drop-out im Schulbereich siehe weiterführend Stamm et al. (2012), Seelinger (2016) und Autorengruppe Bildungsberichterstattung (2018), weiterführend für den Studienbereich Blüthmann et al. (2008), Heublein und Wolter (2011).
} 


\subsection{Modelle zur Erklärung von Drop-out als Weiterbildungsverhalten ${ }^{4}$}

Während Teilnahmestrukturen und Einflussfaktoren in der erwachsenenpädagogischen Teilnahmeforschung mittlerweile gut belegt sind (z. B. im AES), gilt es darüber hinaus, die Gründe für die Beteiligung an und für das Fernbleiben von Weiterbildung zu untersuchen und theoretisch zu erfassen.

Die theoretische Diskussion zum Thema Weiterbildungsteilnahme hat sich erheblich ausdifferenziert (vgl. Büchter 2010; Wittpoth 2018). Zu nennen sind Ansätze auf der Grundlage von Theorien rationaler Entscheidung (z.B. Bolder und Hendrich 2000; Holzer 2004; Reich-Claassen 2010), handlungstheoretische Ansätze (z. B. Rubenson und Salling-Olesen 2007; Haberzeth et al. 2013) und lerntheoretische Ansätze (z.B. Siebert 2003; Faulstich 2013). Eine Verbindung von lern- und habitustheoretischen Ansätzen hat Grotlüschen (2010) mit einer Untersuchung zur Genese und Entwicklung von Weiterbildungsinteressen unternommen. Schließlich sind vor allem habitus- und milieutheoretische Ansätze zu nennen, die sich in der Drop-out-Forschung bereits als tragfähig erwiesen haben (Nairz-Wirth et al. 2014). Hier kommen prägende Bildungserfahrungen und habituelle Grundorientierungen als Regulative in den Blick (vgl. Reich-Claassen et al. 2011).

Die genannten theoretischen Perspektiven können als heuristischer Rahmen für eine Analyse der Veränderung von Lernbegründungen (wie sie im Phänomen Dropout u. U. sichtbar werden) vor dem Hintergrund sich verändernder struktureller und situativer Gegebenheiten und Handlungsanforderungen genutzt werden. Sie zeigen in je unterschiedlicher Fokussierung, dass individuelle Weiterbildungs- und auch Abbruchentscheidungen als ,,subjektiv begründetes Handeln von Akteuren innerhalb von gesellschaftlichen Strukturen“ (Käpplinger et al. 2013, S. 18) und im Kontext von situativen sowie institutionellen Handlungsanforderungen und Gegebenheiten zu verstehen sind. Nicht einzelne subjektive Aspekte sind ausschlaggebend für Entscheidungen, sondern eine „Motivverkopplung“ (Vontobel 1972), innerhalb derer auch Interdependenzen zwischen extrinsischen und intrinsischen Motiven (ReichClaassen 2010), Interessengemengelagen (Grotlüschen 2010) und Emotionen (Gieseke 2007) zu berücksichtigen sind. Diese werden auch durch situative Faktoren beeinflusst.

\subsection{Theoretische Modelle mit Schwerpunkt auf Drop-out}

Für den erwachsenenpädagogischen Bereich forderte Garrison bereits 1987, dass ein theoretischer Forschungsrahmen notwendig sei, um das Phänomen Drop-out zu verstehen. Es gebe nur wenige Versuche, eine systematische Strategie zu erarbeiten und zu viele vergleichende Studien ohne Berücksichtigung der Theoriebildung (vgl. Garrison 1987). Garrison (1988) stellt fünf Variablen (intrinsische und extrinsische Motivation, intrinsische und extrinsische Zwänge sowie Fähigkeiten) als ausschlaggebend für eine Veränderung bzw. eine Stabilität im Verhalten erwachsener Lernender heraus. Hier lässt sich die aus Untersuchungen abgeleitete Differenzie-

\footnotetext{
${ }^{4}$ Die folgenden Ausführungen (3.1., 3.2., 4.) basieren zu Teilen auf dem Antrag zum Forschungsprojekt (vgl. Schmidt-Hertha et al. 2016).
} 
rung von Darkenwald und Gavin (1987) anschließen. Sie sehen eine Diskrepanz zwischen Erwartungen und Erfahrungen als ausschlaggebend für Drop-out an. Dabei sind Teilnahmemotive oder -orientierungen nicht nur multipel, sondern meist weder bewusst noch rational.

Viele der frühen US-amerikanischen Untersuchungen referieren auf die heute auch kritisch hinterfragten Modelle von Boshier (1973) und Tinto (1975). Boshiers Kongruenz-Modell basiert auf psychologischen Annahmen. Er setzt ,self/institution incongruence " für die Weiterbildungsteilnahme voraus, wohingegen bei Drop-out „,intra-self and self/other incongruence“ entstehe (Boshier 1973, S. 255). Besteht keine Passung zwischen den individuellen Bedürfnissen, Erwartungen und Werten sowie den institutionellen Gegebenheiten in Form von wahrgenommenen Haltungen, Werten und Überzeugungen der der Institution angehörigen Personen, erfolgt laut Boshier ein Abbruch. Das Modell von Tinto (1975) betrachtet die Einflussfaktoren auf Drop-out-Verhalten im Rahmen interaktiver universitärer Settings. Es betont die Wichtigkeit der fachlichen und sozialen Integration bei der Bildungsbeteiligung, auf die wiederum andere Faktoren Einfluss haben. Das Modell ist anknüpfungsfähig für verschiedene Bildungsbereiche (für die Schule vgl. Stamm et al. 2012), so auch für die Erwachsenen- und Weiterbildung. Früher noch vernachlässigt, findet in der Betrachtung von Drop-out im gesamten Bildungsbereich zunehmend eine Verschiebung von der individuellen Perspektive hin zur Betrachtung institutioneller Strukturen und Bedingungen als Einflussfaktoren auf Drop-out-Verhalten oder hin zur Integration beider Perspektiven - wie im diesem Artikel zugrundeliegenden Forschungsprojekt - statt.

\section{Forschungsergebnisse zu Drop-out in der Weiterbildung}

Die empirischen sowie theoretischen Betrachtungen zu Drop-out stammen vorwiegend aus den 1970er und 1980er Jahren. Auf Basis von Teilnehmerstatistiken (vgl. z. B. Gruber 1985a; Schrader 1986; Brödel 1996) wurden vor allem Einflussfaktoren im pädagogischen Prozess selbst oder objektive Strukturen (Rahmenbedingungen der Bildungsveranstaltung) untersucht. Damit geht eine angebotsseitige Einengung einher: Der Fokus der empirischen Betrachtung liegt auf „Faktoren, deren Beeinflussung in der Macht der Veranstalter liegt“ (Nuissl und Sutter 1979, S. 129). In den letzten 30 Jahren sind für den deutschsprachigen Raum nur wenige Studien zu Drop-out in einem engen erwachsenenpädagogischen Kontext auffindbar. Ausführlich wurden Drop-outs in Funkkolleg-Begleitkursen untersucht (Lingkost 1996). Im Bereich der Alphabetisierung und Grundbildung von Erwachsenen werden zunehmend Untersuchungen zu Abbrüchen und Unterbrechungen durchgeführt (vgl. u.a. Egloff 2011; Jakschik und Pieniazeka 2011). Auch zu Drop-out im Fernunterricht bzw. in Online-Kursen gibt es eine eigene Forschungstradition, die auch pädagogisch-psychologisch ausgerichtet ist (vgl. Übersicht bei Schmidt 2011).

In der Erwachsenenbildung werden zunehmend Untersuchungen zu Abbrecherquoten rezipiert, die aufgrund der unterschiedlichen Definitionen sowie der Heterogenität der Bildungsmaßnahmen in der Erwachsenenbildung sehr unterschiedlich ausfallen (vgl. zusammenfassend Schmidt 2011; Schröder 1976; Gruber 1985a; 
Schrader 1986). Zumeist schwanken sie zwischen $10 \%$ und $75 \%$ (vgl. Brödel 1996); teilweise sind die Abbrecherquoten jedoch auch nur unzureichend erfasst, wie im AES (vgl. Schmidt 2011). Für den Weiterbildungsbereich sind sowohl die Heterogenität der Bildungsangebote als auch die der Nicht-(mehr-)Teilnehmenden charakteristisch. Während in anderen Bildungsbereichen, wie der Schul- und Berufsausbildung, der Abbruch vor allem benachteiligte Personengruppen betrifft, lässt sich dies für die Weiterbildung nicht eindeutig nachweisen.

Auch wenn auf die oben genannten Befunde zurückgegriffen werden kann, so gibt es derzeit noch keine Studie, die Drop-outs in verschiedenen Bereichen der Weiterbildung umfassend behandelt, sodass hier nach wie vor Forschungsbedarf besteht.

\subsection{Eigene empirische Zugänge}

Im Folgenden werden erste empirische Forschungsergebnisse vorgestellt, welche sich diesem Forschungsdesiderat widmen und innerhalb des noch laufenden DFGgeförderten Projekts „Analyse von Drop-out in der Weiterbildung “5 generiert wurden.

Im Mittelpunkt der Forschungen steht die multiperspektivische Erfassung und Analyse subjektiv relevanter Bedingungen für Drop-out-Entscheidungen. Zugunsten einer Perspektivverschränkung wird die Datenbasis, bestehend aus drei verschiedenen Zugängen, mittels Methoden- und Datentriangulation (vgl. Flick 2004) miteinander kombiniert. Erstens wurden Daten aus der Erwachsenenkohorte des Nationalen Bildungspanels (NEPS) unter Bezugnahme auf multivariate Clusteranalysen und Regressionen zur Identifizierung von Prädiktoren für Weiterbildungsabbrüche und betroffene Gruppen analysiert. Zweitens wurden parallel bundesweit elf Interviews mit Expertinnen und Experten (vgl. Meuser und Nagel 2002) aus allgemeinen, beruflichen, gewerkschaftlichen, politischen und konfessionellen Weiterbildungsverbänden und -einrichtungen geführt. Basierend auf einem von der Grounded Theory geleiteten Zugang werden in einem noch nicht abgeschlossenen dritten Zugang zusätzlich zu dieser Datenbasis ca. 40 problemzentrierte Interviews mit Abbrecherinnen und Abbrechern von Weiterbildungen geführt.

Im Folgenden werden erste Ergebnisse aus den NEPS-Analysen (vgl. Abschn. 4.2), sowie aus den Analysen der Interviews mit den Weiterbildungsexpertinnen und -experten (vgl. Abschn. 4.3) vorgestellt. Im abschließenden Kapitel wird aufgezeigt, welche Erkenntnisse aus diesen beiden empirischen Zugänge für den dritten Zugang (Interviews mit Abbrecherinnen und Abbrechern) gezogen werden können.

\footnotetext{
5 Das Projekt „Analyse von Drop-out in der Weiterbildung (Verbreitung, Einflussfaktoren, Auswirkungen): Entwicklung einer gegenstandsverankerten Theorieperspektive auf Drop-out“" wird von der Deutschen Forschungsgemeinschaft von 10/2017 bis 10/2019 unter folgenden Förderkennzeichen gefördert: SCHM 2391/6-1 und HI 1599/4-1.
} 


\subsection{Erfassung des Phänomens Drop-out in der NEPS-Studie}

Im Nationalen Bildungspanel (NEPS) (vgl. Blossfeld et al. 2011) werden in der Erwachsenenkohorte $(n=17.140)$ Weiterbildungsaktivitäten in mehreren Schleifen mit unterschiedlichen Foki und in Bezug auf die jeweilige Episode erfragt. Als Episode wird dabei ein Zeitraum definiert, in dem die befragte Person einen bestimmten (beruflichen) Status hatte (z. B. erwerbstätig, arbeitslos oder in Elternzeit).

In einem ersten Zugriff wird nach beruflicher Fortbildung in der jeweiligen Episode gefragt, wobei für bis zu drei genannte Weiterbildungsaktivitäten detailliertere Nachfragen gestellt werden und so z. B. auch Weiterbildungsabbrüche erfasst werden. Die Frage „Haben Sie diesen Kurs/Lehrgang vorzeitig abgebrochen oder bis zum Ende teilgenommen?" wird in diesem Teil der Befragung also zunächst nur für maximal drei berufliche Fort- und Weiterbildungen gestellt.

In einem zweiten Zugriff wird nach weiteren Weiterbildungsaktivitäten - explizit auch nach außerberuflichen Weiterbildungen seit dem letzten Interviewzeitpunkt, also unabhängig von einzelnen Episoden - gefragt. Von den so erfassten Weiterbildungsteilnahmen wird ebenso durchgehend erfragt, inwieweit diese abgebrochen oder bis zum Ende besucht wurden. Weitere Details zu der jeweiligen Maßnahme werden aber nur für zwei der besuchten Weiterbildungen erfasst.

In einem dritten Zugriff werden überdies berufliche Weiterbildungsaktivitäten erfasst, die in den Bereich formaler Bildung hineinragen (z. B. Studium, Fach- oder Berufsfachschulausbildung, Umschulungen, Meister- oder Technikerausbildungen, Kurse für den Erwerb von Schweißer-, Taxifahrer-, Gabelstaplerschein). Auch hier wird für alle genannten Bildungsaktivitäten erhoben, ob diese abgebrochen oder bis zum Ende besucht wurden.

Das in NEPS zugrunde gelegte Verständnis von Drop-out scheint auf den ersten Blick an der Teilnahme bzw. Nicht-Teilnahme bis zum Ende des Kurses orientiert. Eine grundsätzliche Nicht-Teilnahme trotz vorangegangener Anmeldung zu einem Kurs - also ein Abbruch vor dem ersten Weiterbildungstermin - wird mit dem NEPS-Instrumentarium nicht erfasst. Interessanterweise bleibt die entsprechende Frage nach Abbrüchen auch unverändert, wenn es um formale Weiterbildungen geht - also Bildungsaktivitäten, die auf den erfolgreichen Erwerb einer Lizenz oder eines Zertifikats ausgerichtet sind und in der Regel entsprechende Abschlussprüfungen implizieren. Man hätte hier Drop-out auch an das Bestehen der Prüfung bzw. den Erwerb des jeweiligen Zertifikats knüpfen können, was aber in NEPS anders operationalisiert wurde. Nach dem Erreichen persönlicher oder curricularer Bildungsziele wird hierbei nicht gefragt, ebenso nicht nach der Teilnahme an oder dem Erfolg bei mit den jeweiligen Bildungsmaßnahmen verbundenen Prüfungen. Auch werden Regelmäßigkeit der Teilnahme oder Gründe für einen Weiterbildungsabbruch nicht erhoben. Den Befragten wird somit suggeriert, dass die Teilnahme „bis zum Ende“ als einzige Alternative dem Weiterbildungsabbruch gegenübersteht.

Die Auswertung der Daten in Bezug auf Drop-out ist nicht nur aufgrund der unterschiedlichen Informationen, die zur jeweiligen Weiterbildung zur Verfügung stehen, herausfordernd, sondern durch das Fehlen von Informationen zu den Gründen für einen Weiterbildungsabbruch zudem begrenzt aussagekräftig, wenn es um die Frage nach der Bedeutung von Drop-outs für die Lernenden selbst geht. 
Tab. 1 Prädiktoren für Weiterbildungsabbruch: lineare Regressionsanalyse $(n=17.140)$

\begin{tabular}{|c|c|c|c|c|c|}
\hline & \multirow[t]{2}{*}{$\begin{array}{l}\text { Ausprägungen der } \\
\text { Variablen }\end{array}$} & \multicolumn{2}{|c|}{$\begin{array}{l}\text { nichtstandardisierte Koeffizi- } \\
\text { enten }\end{array}$} & \multirow{2}{*}{$\begin{array}{l}\text { standardisierte } \\
\text { Koeffizienten } \\
\text { Beta }\end{array}$} & \multirow[t]{2}{*}{ Sig } \\
\hline & & $\begin{array}{l}\text { Regressions- } \\
\text { koeffizient B }\end{array}$ & $\begin{array}{l}\text { Standard- } \\
\text { fehler }\end{array}$ & & \\
\hline (Konstante) & - & 1,990 & 0,004 & - & 0,000 \\
\hline Geschlecht & $\begin{array}{l}1=\text { männlich } \\
2=\text { weiblich }\end{array}$ & $-0,004$ & 0,001 & $-0,018$ & 0,000 \\
\hline $\begin{array}{l}\text { Muttersprache: } \\
\text { deutsch }\end{array}$ & $\begin{array}{l}1=\mathrm{ja} \\
2=\text { nein }\end{array}$ & $-0,013$ & 0,002 & $-0,024$ & 0,000 \\
\hline $\begin{array}{l}\text { Haushalts-Netto- } \\
\text { einkommen }\end{array}$ & metrisch & 0,003 & 0,000 & 0,044 & 0,000 \\
\hline $\begin{array}{l}\text { Kursdauer insge- } \\
\text { samt (Stunden) }\end{array}$ & metrisch & 0,000 & 0,000 & $-0,069$ & 0,000 \\
\hline $\begin{array}{l}\text { Bildungsabschluss } \\
\text { Mutter (CAS- } \\
\text { MIN) }\end{array}$ & $\begin{array}{l}0=\text { kein Abschl. } \\
8=\text { Uni-abschl }^{\text {a }}\end{array}$ & $-0,001$ & 0,000 & $-0,011$ & 0,023 \\
\hline
\end{tabular}

$A V$ WB-Abbruch

$\mathrm{R}^{2}=0,009$

${ }^{a}$ vgl. Lechert et al. (2006), S. 5

Es ist grundsätzlich davon auszugehen, dass der Anteil abgebrochener Maßnahmen in den NEPS-Daten unterschätzt wird, da die Eingangsfrage nach besuchten Lehrgängen und Kursen u. U. dazu führt, dass gerade frühzeitig abgebrochene Weiterbildungen von den Befragten nicht thematisiert werden. Dennoch geben die Daten wertvolle Hinweise auf häufige Abbruchskonstellationen. So weisen deskriptive Auswertungen der ungewichteten Personendaten über alle zum Auswertungszeitpunkt vorliegenden acht Erhebungswellen hinweg darauf hin, dass im außerberuflichen Bereich Sprachkurse besonders häufig abgebrochen werden, was auch mit deren durchschnittlich längeren Dauer zu tun haben kann. Mit der Dauer einer Weiterbildungsmaßnahme steigt auch die Wahrscheinlichkeit eines Abbruchs, und abgebrochene berufliche Weiterbildungen dauern im Durchschnitt fast doppelt so lange wie nicht abgebrochene (96,4h vs. 51,2 h).

Anhand unterschiedlicher Regressionsmodelle, die hier nicht vollständig dargestellt werden können, wurde der Einfluss unterschiedlicher soziodemografischer Variablen auf Weiterbildungsabbrüche (hier als dichotome Variable) geprüft. Da die geringe Fallzahl von Abbrüchen (738 Abbrüche von 697 Personen) nur eine begrenzte Anzahl von unabhängigen Variablen in einem Regressionsmodell zulässt, wurden in das hier vorgestellte Modell nur diejenigen Variablen einbezogen, die sich in verschiedenen Regressionsmodellen als relevante Prädiktoren für Abbrüche erwiesen hatten. Andere Variablen (z. B. Alter, Schulbildung, Erwerbsstatus etc.) standen bei Berücksichtigung der in Tab. 1 aufgeführten Variablen in keinem signifikanten Zusammenhang mit der abhängigen Variablen. Allerdings verweist die äußerst geringe Varianzaufklärung auch darauf, dass der Effekt der im Modell berücksichtigten Variablen insgesamt eher gering ist.

Insgesamt lässt sich zeigen, dass Personen mit geringerem Einkommen und mit Migrationshintergrund Weiterbildungen häufiger abbrechen als andere, und Frauen 
ein höheres Abbruchrisiko als Männer haben. Außerdem ließ sich auch ein moderater Effekt des Bildungsabschlusses der Mutter nachweisen (siehe Tab. 1).

\subsection{Perspektive von Expertinnen und Experten auf das Phänomen Drop-out}

Leitfadengestützte Experteninterviews ermöglichen in diesem Forschungsprojekt den Einbezug einer institutionellen Ebene, die in der Theoriediskussion als relevante Betrachtungsverschiebung von Drop-out ersichtlich wird. Hinsichtlich der Auswahl der elf Expertinnen und Experten wurde darauf geachtet, dass sie als Repräsentantinnen und Repräsentanten möglichst die Heterogenität der Weiterbildungsbereiche abbilden. Dementsprechend wurden sie aus allgemein, beruflich, gewerkschaftlich, politisch und konfessionell bildenden Einrichtungen rekrutiert. Diese Expertinnen und Experten fungieren als Funktionsträger und verfügen damit über einen privilegierten Zugang zu den jeweiligen institutionellen Kontexten sowie zu Informationen über Personengruppen oder Entscheidungsprozesse. Zu Gegenständen der Interviews wurden nicht nur deren Aufgaben, Tätigkeiten und Zuständigkeiten, sondern vor allem auch deren Wissensbestände, die aus diesen Erfahrungen hervorgehen (vgl. Meuser und Nagel 2002).

In der Anlage der Leitfadeninterviews wurde darauf geachtet, dass nach der Vorstellung der Expertinnen und Experten zunächst eine Initiierung einer selbstläufigen Sachverhaltsdarstellung erfolgte. Anschließend folgte eine Phase des immanenten Nachfragens zum Erzählten sowie eine Phase des exmanenten Nachfragens zu verschiedenen thematischen Schwerpunkten, die aus vorheriger Literaturrecherche zu Drop-out bzw. Weiterbildungsabbruch und dem aktuellen Forschungsstand hervorgegangen sind, z. B. zur Erfassung von Drop-out in den Einrichtungen, zu wahrgenommenen Einflussfaktoren und Abbruchgründen und zum präventiven sowie situativen Umgang mit Drop-outs.

Die so ermittelten Perspektiven auf das Phänomen Drop-out in der Weiterbildung wurde mittels strukturierender Inhaltsanalyse durchgeführt (vgl. Mayring 2015). Zudem konnten durch eine komparative Analyse der Interviews ,überindividuellgemeinsame Wissensbestände“ (Meuser und Nagel 2011, S. 58) identifiziert werden.

Im Folgenden werden vier Kategorien von Drop-out (Definitionseinheit, formale sowie non-formale Angebotsform, Akteure der Herbeiführung, zeitlicher Verlauf) vorgestellt, die in der Auswertung der Interviews mit Expertinnen und Experten hinsichtlich einer Begriffsbestimmung des Phänomens Drop-out herausgearbeitet wurden.

\subsubsection{Definitionseinheit}

Die Ergebnisse aus den Interviews lassen Rückschlüsse darauf zu, was von den Expertinnen und Experten als Drop-out identifiziert wird, was also eine (Definitions-)Einheit von Drop-out sein kann. Darunter wird (1) durchgängig der Abbruch eines Kurses gefasst, der in Form einer Einzelveranstaltung oder eines Kurses mit mehreren Einheiten zu verschiedenen Zeitpunkten stattfinden kann. Ferner werden auch (2) Abbrüche bzw. die Nicht-Teilnahme an aufeinander aufbauenden 
Kursen (wie Sprachkurse) und (3) das Herausfallen aus dem Weiterbildungskontext bzw. des lebenslangen Lernens darunter verstanden.

Die interviewten Praktikerinnen und Praktiker beziehen sich also auf Drop-outVarianten, wie sie auch bereits bei Brödel (1996) und Schmidt (2011) behandelt wurden.

Während Variante 1, auf der auch der Fokus bisheriger Literatur zu Drop-out bzw. Abbrüchen liegt (vgl. Brödel 1996; Djafari et al. 1989; Gruber 1985b; Lingkost 1996, Schröder 1976; Wolter 1987 etc.), von Expertinnen und Experten aller Weiterbildungsbereiche thematisiert wird, wird Variante 2 vornehmlich im Bereich der allgemeinen und konfessionellen Weiterbildung benannt. Anknüpfungspunkte in der Erwachsenenbildung bietet hier am ehesten die Forschung zur Nichtteilnahme an Weiterbildung, jedoch nicht mit dem Fokus des Abbruchs, sondern des NichtBeginnens (vgl. Wittpoth 2018; von Hippel et al. 2018). Variante 3 beruht auf einer Einzelnennung eines Experten aus der allgemeinen Weiterbildung. In der Erwachsenenbildungsforschung wird dies somit nicht repräsentativ unter dem Begriff ,Dropout" behandelt.

\subsubsection{Drop-out in der formalen und non-formalen Weiterbildung}

Drop-out wird dort thematisiert, wo es formale Vorgaben (wie Curricula und festgelegte Prüfungszeiträume) sowie formale Abschlüsse gibt, folglich also in der (1) formalen Bildung. Eine besondere Variante stellt hier jene dar, die zusätzlich an Belohnung und Sanktionen gebunden ist, z. B. bei staatlicher Förderung durch das Jobcenter. In diesem Fall ist es nicht nur möglich, dass die Person selbst die Weiterbildung vorzeitig beendet, sondern auch, dass die Förderinstitution den Dropout der Teilnehmenden vollzieht, z. B. wenn sie die Rahmenbedingungen der Finanzierung nicht gewährleistet sieht. Diese Variante 1 ist ein Thema in Weiterbildungseinrichtungen aus dem Kontext der beruflichen Bildung, der Alphabetisierung und der Integrationskurse. Zusätzlich wird Drop-out im Bereich der (2) non-formalen Bildung beobachtet, z. B. in Kursen zum Stressmanagement.

Diese Ergebnisse reihen sich in die bisherigen Forschungsergebnisse zu Drop-out ein, die hinsichtlich der Erwachsenenbildung punktuell sind, aber in ihren Ausführungen konsequent den formalen und non-formalen Bildungskontext abbilden. Der von den Befragten nicht thematisierte (3) informelle Weiterbildungsbereich wird bisher auch in der Drop-out-Literatur nicht bearbeitet.

\subsubsection{Drop-out aus Akteursperspektive}

Ein Weiterbildungsabbruch kann grundsätzlich von zwei Seiten aus vorgenommen werden: (A) Selbst herbeigeführte Abbrüche werden in den Interviews am häufigsten seitens Expertinnen und Experten aller Weiterbildungsbereiche thematisiert. Die Entscheidung dafür liegt i.d.R. bei den Teilnehmenden selbst und fußt auf vielfältigen individuellen, institutionellen, (sozio-)strukturellen und situativen Gründen sowie Ursachen. (B) Durch Institutionen herbeigeführte Drop-outs zeigen sich als vorzeitige Beendigung der Weiterbildung seitens staatlicher Fördereinrichtungen (wie der Agentur für Arbeit) oder des Weiterbildungsträgers. Gründe dafür können 
z.B. eine Arbeitsaufnahme zum Ende der Weiterbildung sowie die Annahme sein, dass das Bildungsziel aufgrund zu hoher Fehlzeiten nicht erreicht werden kann. Auch ethische Überlegungen, Bedenken gegenüber der Leistungsfähigkeit der Teilnehmenden oder Konsequenzen in Folge von Täuschungsversuchen können eine Rolle spielen. Ferner kann ein Abbruch ausschließlich formal/bürokratisch vorliegen, z.B. wenn Teilnehmende in einen anderen Umschulungsjahrgang wechseln (u. a. nach einer Schwangerschaft). Diese Art der Beendigung spielt in der beruflichen Weiterbildung eine Rolle. Des Weiteren kann ein Drop-out durch die Weiterbildungseinrichtung vollzogen werden, ohne dass diese Entscheidung in Verbindung mit dem Verhalten von Teilnehmenden direkt zusammenhängt, z. B. aufgrund von Insolvenz, Nichterreichen der Mindestteilnehmerzahl pro Kurs und fehlendem Lehrpersonal. Diese Form des Abbruchs findet sich in beruflicher und außerberuflicher Weiterbildung gleichermaßen.

Ein Graubereich in dieser Logik ist z. B. dort zu finden, wo staatliche Einrichtungen, wie das Jobcenter, die Agentur für Arbeit oder die Deutsche Rentenversicherung, die Finanzierung der Weiterbildung aufgrund zu hoher Fehlzeiten streicht, diese jedoch möglicherweise bewusst durch die Teilnehmenden herbeigeführt wurden. Der Drop-out wäre dann nicht eindeutig von der Institution initiiert, sondern läge auch im Verantwortungsbereich der Teilnehmenden.

Bestehende Forschungsliteratur betrachtet vornehmlich die Perspektive des selbst herbeigeführten Drop-outs; von den Institutionen veranlasste Drop-outs werden in der einschlägigen Literatur nicht in den Blick genommen. Anleihen könnten hier in der Drop-out-Forschung im Schulkontext gemacht werden, wo Stamm et al. (2012) Abbrüche seitens der Institution Schule als „Push-out“ kennzeichnet und vom „Pull-out“ als freiwilligen Abbruch seitens der Schülerinnen und Schüler sowie zum „Fade-out“ als allmähliches Ausklinken im Sinne eines länger andauernden Prozesses abgrenzt. Der Begriff des „Push-outs“ spiegelt durchaus den durch die Institution herbeigeführten „Drop-out“ wider. Hierdurch wird deutlich, dass Sanktionierung und Selektion nicht nur im tertiären, sondern durchaus auch im quartären Bereich wirken, obwohl diesem oft eine höhere Freiwilligkeit in der Teilnahme und Gestaltung der Lernprozesse zugesprochen wird.

\subsubsection{Drop-out aus einem Kurs unter zeitlicher Perspektive}

Hinsichtlich einer zeitlichen Verortung von Drop-out geht hervor, dass Abbrüche zu verschiedenen Zeitpunkten des Kursgeschehens identifiziert werden (siehe Abb. 1).

Nur selten wird seitens der Interviewten der Begriff „Drop-out“ als Nicht-Teilnahme an einem Folgekurs, der sich an den Stellen A bis C bzw. D vollzieht, verstanden. Der Großteil der Abbrüche lässt sich nach diesen an den Punkten D bis F bzw. H verorten. Hierbei wird von ihnen zwischen einem dauerhaften und einem periodischen Fernbleiben der Teilnehmenden unterschieden.

Ersteres wird in jedem Fall als Abbruch und letzteres - je nach Weiterbildungskontext - als Abbruch oder Unterbrechung gewertet. Das hängt damit zusammen, dass ein periodisches Fernbleiben in der beruflichen Bildung Anlass zum Beenden einer Bildungsveranstaltung seitens staatlicher Fördereinrichtungen sein kann, was zu einem Drop-out führt, in der allgemeinen Weiterbildung hingegen jedoch ledig- 
Abb. 1 Drop-out im zeitlichen Verlauf

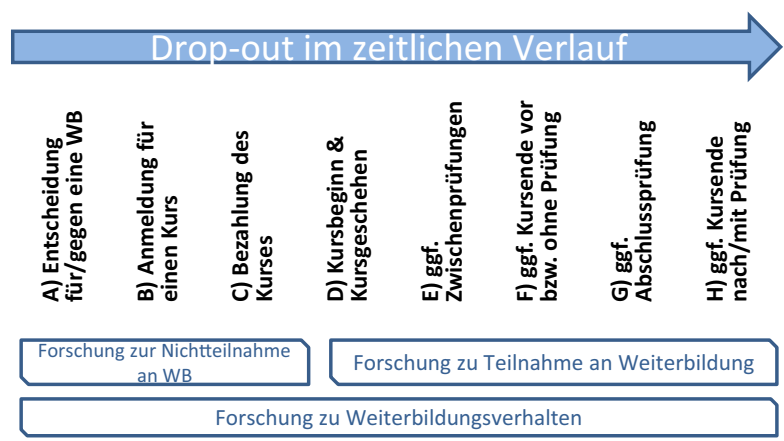

lich formale Konsequenzen wie das Nichtausstellen einer Teilnahmebescheinigung nach sich ziehen kann.

Umgekehrt ist auch der Verbleib bis zum Kursende mit Blick auf mögliche Leistungsnachweise uneindeutig, da ein Kurs (a) ohne eine Abschlussprüfung sowie (b) vor den Abschlussprüfungen enden kann oder gar (c) die Prüfungen dem Kursende vorgelagert sind. Die Nichtteilnahme an bzw. das Nichtbestehen einer Abschlussprüfung am Ende des Kurses ist nicht zwingend ein Drop-out, es sei denn, diese ist obligatorisch. Ein Abbruch kann folglich erst dann identifiziert werden, wenn es keine Prüfungsmöglichkeit mehr gibt bzw. der Prüfungsanspruch erloschen ist.

Expertinnen und Experten, die diese Prüfungsperspektiven an den Stellen G bis $\mathrm{H}$ einnehmen, sind jene aus dem Bereich der beruflichen Bildung, wohingegen die Expertinnen und Experten, die von Drop-outs an den Stellen D bis F sprechen, in der Regel die aus dem Bereich der außerberuflichen Bildung sind. Diese Perspektive deckt sich mit Ausführungen bisheriger Drop-out-Literatur: Fokussiert werden hier Drop-outs, die zu späteren Zeitpunkten im Kursverlauf (D bis H) identifiziert werden.

\section{Fazit und Ausblick}

Die oben ausgeführten Ergebnisse aus den NEPS-Daten sowie der Befragung von Expertinnen und Experten können dazu dienen, das Phänomen Drop-out in der Erwachsenenbildung begrifflich zu schärfen und über das Forschungsprojekt hinaus näher zu charakterisieren, vor allem in Form von Kategorien, anhand derer (einzeln und in ihren Kombinationen betrachtet) Konstellationen von Drop-out beschrieben werden können. So lassen sich mit den NEPS-Daten typische Abbruchkonstellationen identifizieren, die sich aus der Kombination von soziodemografischen und angebotsbezogenen Kategorien ergeben. Die aus der Befragung von Expertinnen und Experten gewonnenen Kategorien können ebenfalls dazu genutzt werden, Drop-outKonstellationen z.B. anhand der Kombination von Kategorien zur zeitlichen Verortung und Herbeiführung von Drop-out zu beschreiben. Die im Projekt geführten Interviews können wiederum die individuellen, institutionellen, (sozio-)strukturellen und situativen Gründe für und Folgen von Drop-outs beleuchten, da sie triangulativ mit den Konstellationen, in denen sie auftreten, verknüpft und ausgewertet wer- 
den. Die so gewonnenen Ergebnisse schließen an die Theorien und Modelle zum Weiterbildungsverhalten an.

Forschungsdesiderate bestehen darin, Drop-outs in der quantitativen Beteiligungsforschung detaillierter zu erfassen sowie frühe Formen des Drop-outs (z. B. jene, die noch vor Kursbeginn erfolgen) zu untersuchen. Dies wäre unter der Fragestellung interessant, wie Weiterbildungsentscheidungen zustande kommen. Anregend wäre dies auch für die Erwachsenenbildungspraxis, in der sich kurzfristige Absagen bzw. Rücktritte vor Kursbeginn häufen. Hieran anschließend wäre eine genauere Untersuchung interessant, wie die Erwachsenenbildungseinrichtungen mit Drop-out umgehen, da Drop-out eine Herausforderung für die professionelle didaktische Gestaltung von Planenden wie Lehrenden ist.

Aus der Perspektive der professionellen Antinomien (vgl. Helsper 2002; für die Erwachsenenbildung von Hippel 2011) spitzt sich für die professionellen Erwachsenenbildnerinnen und -bildner - aber auch für die Teilnehmenden - im Drop-out die Ungewissheitsantinomie zu. Erwachsenenbildung will - als professionelle Praxis nach Oevermann (1996) interpretiert - stellvertretend deutend Autonomiepotenziale der Lebenspraxis der Teilnehmenden stärken. Es geht darum, durch die stellvertretende Deutung etwas zu ermöglichen, was noch nicht ist (Bildung, Kompetenzen etc.). Die Ungewissheitsantinomie meint hier, dass Erwachsenenbildnerinnen und -bildner Vermittlungsversprechen machen müssen bei gleichzeitig struktureller Ungewissheit, ob gemeinsam Bildungsziele erreicht werden können (da das Erreichen nicht völlig in den Händen der Professionellen liegt).

Der Begriff „Drop-out“ kann in verschiedenen Konstellationen damit sowohl Ausdruck legitimen freiwilligen Bildungsverhaltens wie auch didaktisches Problem sein. In jedem Fall wirft ein Drop-out immer auch Fragen nach gesellschaftlicher Teilhabe und der Gestaltung von Bildungsbiografien auf (vgl. auch Schrader 1986; Brödel 1996).

Open Access Dieser Artikel wird unter der Creative Commons Namensnennung 4.0 International Lizenz (http://creativecommons.org/licenses/by/4.0/deed.de) veröffentlicht, welche die Nutzung, Vervielfältigung, Bearbeitung, Verbreitung und Wiedergabe in jeglichem Medium und Format erlaubt, sofern Sie den/die ursprünglichen Autor(en) und die Quelle ordnungsgemäß nennen, einen Link zur Creative Commons Lizenz beifügen und angeben, ob Änderungen vorgenommen wurden.

\section{Literatur}

Autorengruppe Bildungsberichterstattung (2018). Bildung in Deutschland 2018. Ein indikatorengestützter Bericht mit einer Analyse zu Wirkungen und Erträgen von Bildung. https://www.bildungsbericht. de/de/bildungsberichte-seit-2006/bildungsbericht-2018/pdf-bildungsbericht-2018/bildungsbericht2018.pdf. Zugegriffen: 17. Mai 2018.

Blossfeld, H.-P., Rossbach, H.-G., \& von Maurice, J. (Hrsg.). (2011). Education as a lifelong process: The German National Educational Panel Study (NEPS). Wiesbaden: VS.

Blüthmann, I., Lepa, St , \& Thiel, F. (2008). Studienabbruch und -wechsel in den neuen Bachelorstudiengängen. Untersuchung und Analyse von Abbruchgründen. Zeitschrift für Erziehungswissenschaft, 11(3), 406-429.

Bolder, A., \& Hendrich, W. (2000). Fremde Bildungswelten. Alternative Strategien lebenslangen Lernens. Opladen: Leske + Budrich.

Boshier, R. (1973). Educational participation and dropout: a theoretical model. Adult Educational Quarterly, 23(4), 255-282.

Brödel, R. (1996). Dropout - Kursabbruch in der Erwachsenenbildung. Unterrichtswissenschaft: Zeitschrift für Lernforschung, 24(1), 21-31. 
Büchter, K. (2010). Berufliche Weiterbildungsbeteiligung - theoretische und historiographische Zugänge. http://www.bwpat.de/content/uploads/media/buechter_bwpat19.pdf. Zugegriffen: 23. Jan. 2014.

Darkenwald, G. G., \& Gavin, W. J. (1987). Dropout as a function of discrepancies between expectations and actual experiences of the classroom social environment. Adult Education Quarterly, 37(3), 152-163.

Djafari, N., Kade, S., Federwisch, J., \& Klein, R. (1989). Umschulungsabbruch. Reihe: Praxishilfen für die Umschulung, Heft 4. Frankfurt/Main: Pädagogische Arbeitsstelle des Deutschen VolkshochschulVerbandes e. V.

Egloff, B. (2011). Kurs ohne Übergang? Teilnehmerinnen und Teilnehmer an Alphabetisierungskursen. In B. Egloff \& A. Grotlüschen (Hrsg.), Forschen im Feld der Alphabetisierung und Grundbildung. Ein Werkstattbuch (S. 175-190). Münster: Waxmann.

Faulstich, P. (2013). Menschliches Lernen. Eine kritisch-pragmatische Lerntheorie. Bielefeld: transcript.

Flick, U. (2004). Triangulation - Eine Einführung. Wiesbaden: VS.

Garrison, D. R. (1987). Researching dropout in distance education. Distance Education, 8(1), 95-101.

Garrison, D.R. (1988). A deductively derived and empirically confirmed structure of factors associated with dropout in adult education. Adult Education Quarterly, 38(4), 199-210.

Gaupp, N., Geier, B., Lex, T., \& Reißig, B. (2011). Wege in Ausbildungslosigkeit. Determinanten misslingender Übergänge in Ausbildung von Jugendlichen mit Hauptschulbildung. Zeitschrift für Pädagogik, 57(2), 173-186. https://www.pedocs.de/volltexte/2014/8713/pdf/ZfPaed_2_2011_Gaupp_et_al_ Wege_in_Ausbildungslosigkeit.pdf.

Gieseke, W. (2007). Lebenslanges Lernen und Emotionen. Wirkungen von Emotionen auf Bildungsprozesse aus beziehungstheoretischer Perspektive. Bielefeld: wbv.

Grotlüschen, A. (2010). Erneuerung der Interessentheorie. Die Genese von Interessen an Erwachsenenund Weiterbildung. Wiesbaden: Springer VS.

Gruber, Chr (1985a). Determinanten des Kursabbruchs in der Erwachsenenbildung. Linz: BWP.

Gruber, Chr (1985b). Kursabbruch von Teilnehmern in der Volkshochschule. Die österreichische Volkshochschule, 36(135), 5-8.

Haberzeth, E., Käpplinger, B., \& Kulmus, C. (2013). Geld und Bildungsentscheidungen - themen- und lebenslaufbezogene Teilnahmeeffekte des Bildungsschecks Brandenburg. In B. Käpplinger, R. Klein \& E. Haberzeth (Hrsg.), Weiterbildungsgutscheine (S. 211-238). Bielefeld: wbv.

Helsper, W. (2002). Lehrerprofessionalität als antinomische Handlungsstruktur. In M. Kraul, W. Marotzki \& C. Schweppe (Hrsg.), Biographie und Profession (S. 64-102). Bad Heilbrunn: Klinkhardt.

Heublein, U., \& Wolter, A. (2011). Studienabbruch in Deutschland. Definition, Häufigkeit, Ursachen, Maßnahmen. Zeitschrift für Pädagogik, 57(2), 214-236.

von Hippel, A. (2011). Programmplanungshandeln im Spannungsfeld heterogener Erwartungen: ein Ansatz zur Differenzierung von Widerspruchskonstellationen und professionellen Antinomien. REPORT - Zeitschrift für Weiterbildungsforschung, 34(1), 45-57.

von Hippel, A., Tippelt, R., \& Gebrande, J. (2018). Adressaten-, Teilnehmer- und Zielgruppenforschung in der Erwachsenenbildung. In R. Tippelt \& A. von Hippel (Hrsg.), Handbuch Erwachsenenbildung/ Weiterbildung 6.. Aufl. (Bd. 2, S. 1131-1147). Wiesbaden: Springer VS.

Holzer, D. (2004). Widerstand gegen Weiterbildung. Weiterbildungsabstinenz und die Forderung nach lebenslangem Lernen. Wien: Lit.

Jakschik, R., \& PIeniazeka, J. (2011). Über die Beweggründe für Abbrüche von zweitsprachlichen Alphabetisierungskursen und Anregungen für mögliche Gegenmaßnahmen. In Projektträger im Deutschen Zentrum für Luft- und Raumfahrt e. V. (Hrsg.), Lernprozesse in der Alphabetisierung und Grundbildung Erwachsener. Diagnostik, Vermittlung, Professionalisierung (S. 253-266). Bielefeld: wbv.

Käpplinger, B., Kulmus, C., \& Haberzeth, E. (2013). Weiterbildungsbeteiligung. Anforderungen an eine Arbeitsversicherung. WISO-Diskurs. Bonn: Friedrich-Ebert-Stiftung. library.fes.de/pdf-files/ wiso/09852.pdf. Zugegriffen 03.04.2019

Lechert, Y., Schroedter, J., \& Lüttinger, P. (2006). Die Umsetzung der Bildungsklassifikation CASMIN für die Volkszählung 1970, die Mikrozensus-Zusatzerhebung 1971 und die Mikrozensen 1976-2004. ZUMA-Methodenbericht 2006/12.

Lingkost, A. (1996). Teilnahme und Nichtteilnahme an Funkkolleg-Begleitkursen: Eine qualitative Studie zur Drop-out-Forschung. Analysen für Erwachsenenbildung. Frankfurt am Main: Deutsches Institut für Erwachsenenbildung. http://www.die-frankfurt.de/esprid/dokumente/doc-1996/lingkost96_ 01.pdf. Zugegriffen: 26. Aug. 2017.

Mayring, Ph (2015). Qualitative Inhaltsanalyse. In U. Flick, E. von Kardoff \& I. Steinke (Hrsg.), Qualitative Forschung. Ein Handbuch (11. Aufl. S. 468-475). Reinbek bei Hamburg: Rowohlt. 
Meuser, M., \& Nagel, U. (2002). ExpertInneninterviews - vielfach erprobt, wenig bedacht. Ein Beitrag zur qualitativen Methodendiskussion. In A. Bogner, B. Littig \& W. Menz (Hrsg.), Das Experteninterview. Theorie, Methode, Anwendung (S. 71-93). Wiesbaden: Springer.

Meuser, M., \& Nagel, U. (2011). Experteninterview. In R. Bohnsack, W. Marotzki \& M. Meuser (Hrsg.), Hauptbegriffe Qualitativer Sozialforschung (Bd. 3, S. 57-58). Opladen \& Farmington Hills: Barbara Budrich.

Nairz-Wirth, E., Gitschthaler, M., \& Feldmann, K. (2014). Quo Vadis Bildung? Eine qualitative Längsschnittstudie zum Habitus von Early School Leavers. Wien. https:/www.wien.gv.at/wirtschaft/ standort/pdf/quovadis-bildung-2014.pdf. Zugegriffen: 21. März 2016.

Nuissl, E. (2010). Dropout. In R. Arnold, S. Nolda \& E. Nuissl (Hrsg.), Wörterbuch Erwachsenenbildung (2. Aufl. S. 69-70). Bad Heilbrunn: Klinkhardt.

Nuissl, E., \& Sutter, H. (1979). Dropout in der Weiterbildung. Eine Literaturexpertise empirischer Untersuchungen. Arbeitsgruppe für empirische Bildungsforschung. Heidelberg: Esprint.

Oevermann, U. (1996). Theoretische Skizze einer revidierten Theorie professionalisierten Handelns. In A. Combe \& W.W. Helsper (Hrsg.), Pädagogische Professionalität (S. 70-182). Frankfurt a.M.: Suhrkamp.

Reich-Claassen, J. (2010). Warum Erwachsene (nicht) an Weiterbildungsveranstaltungen partizipieren. Einstellungen und prägende Bildungserfahrungen als Regulative des Weiterbildungsverhaltens. Münster: LIT.

Reich-Claassen, J., von Hippel, A., \& Tippelt, R. (2011). Zielgruppenkonstruktion(en) auf Basis des Milieumodells? Ein kritischer Blick auf Milieuforschung und milieuorientierte Bildungsarbeit. In H. Herzberg \& E. Kammler (Hrsg.), Biographie und Gesellschaft (S. 101-118). Frankfurt a.M.: Campus.

Rubenson, K., \& Salling-Olesen, H. (2007). Theorizing participation in adult education and training. https://rucforsk.ruc.dk/ws/files/47046733/learning_motivation_and_participation_KR_HSO_Link_ ping2007.doc. Zugegriffen: 4. Apr. 2019.

Schmidt, B. (2011). Dropout in der Erwachsenenbildung. Zeitschrift für Pädagogik, 57(2), $203-213$.

Schmidt-Hertha, B., von Hippel, A., Stimm, M., \& Kulmus, C. (2016). Analyse von Dropout in der Weiterbildung (Verbreitung, Einflussfaktoren, Auswirkungen): Entwicklung einer gegenstandsverankerten Theorieperspektive auf Drop-out. Bewilligter DFG-Projektantrag. Tübingen: Berlin.

Schrader, J. (1986). Teilnahmeverhalten und Teilnehmerschwund in Volkshochschulkursen. Frankfurt am Main: Pädagogische Arbeitsstelle des Deutschen Volkshochschul-Verbandes.

Schröder, H. (1976). Teilnahme und Teilnehmerschwund als Problem der Erwachsenenbildung. Eine empirische Untersuchung zur Soziologie der Erwachsenenbildung. Stuttgart: Klett.

Seelinger, S. (2016). Schulabsentismus und Schuldropout. Fallanalysen zur Erfassung eines Phänomens. Wiesbaden: Springer.

Siebert, H. (2003). Didaktisches Handeln in der Erwachsenenbildung. Didaktik aus konstruktivistischer Sicht. München: Luchterhand.

Stamm, M., Holzinger-Neulinger, M., \& Suter, P. (2012). Schulabbrecher in unserem Bildungssystem. Wiesbaden: Springer VS.

Tenorth, H.-E., \& Tippelt, R. (2007). BELTZ Lexikon Pädagogik. Weinheim, Basel: Beltz.

Tinto, V. (1975). Dropout from higher education; a theoretical synthesis of recent research. Review of Educational Research, 45(1), 89-125.

Vontobel, J. (1972). Über den Erfolg in der Erwachsenenbildung. Empirische Basisstudie zum Problem der Erfassung des Bildungserfolgs. Braunschweig: Westermann.

Wittpoth, J. (2018). Beteiligungsregulation in der Weiterbildung. In I. R. Tippelt \& A. von Hippel (Hrsg.), Handbuch Erwachsenenbildung/Weiterbildung 6. Aufl. (Bd. 2, S. 1149-1172). Wiesbaden: Springer VS.

Wolter, B. (1987). Kursabbruch. Erfahrungen und Konsequenzen. Informationen Alphabetisierung und elementare Qualifikationen, 1987(4), 13-14.

Publisher's Note Springer Nature remains neutral with regard to jurisdictional claims in published maps and institutional affiliations. 